\title{
Adhäsionen durch Fehlprogrammierung von Stammzellen?
}

Formieren sich Adhäsionen aufgrund von falscher Programmierung von Stammzellen?

\section{Einführung}

Trotz der jüngsten Fortschritte in minimalinvasiven Techniken und prophylaktischen Mitteln bleiben postoperative adhäsionsbedingte Komplikationen wie Subfertilität, Dyspareunie, chronischer Schmerz und Darmverschluss die häufigsten Folgeerscheinungen einer abdominellen Operation. Bis zu 90\% der Patienten entwickeln nach der Operation Adhäsionen in einem unvorhersehbaren Grad [1]. Daher sind weitere Strategien zur Adhäsionsminderung notwendig, um diese iatrogen induzierte Belastung von Patienten und Krankenversicherungssystemen zu reduzieren und die hohe Rate an adhäsionsbedingten Krankenhauseinweisungen zu minimieren [2].

\section{Physiopathologie}

Physiopathologisch gesehen ist die Adhäsionsbildung ein übermäßiger Heilungsprozess mit nachfolgender Vernarbung, der auftritt, wenn die normale Heilung einschließlich Fibrinablagerung nicht durch Fibrinolyse abgeschlossen wird, sodass eine Heilung im Sinne einer Restitutio ad integrum nicht möglich ist [3]. Bei der Erforschung epithelialer Brandwunden ist bekannt, dass die verantwortlichen reparierenden Stammzellen während des Regenerationsprozesses mithilfe ihrer Untereinheit Beta-CR zu den Läsionen wandern. Durch Arzneimittelmodulation dieser Vorläuferzellen konnte bereits eine Heilung ohne Narbenbildung erreicht werden [4].

\section{Experimentelle Studie}

Unsere erste Pilotstudie, bei der Biopsien normaler peritonealer Auskleidung und Adhäsionen bei 16 Patienten mit abdominalen Eingriffen ausgewertet wurden, zeigte eine Gesamtpräsenz von CD-90-po- sitiven Stammzellen, wie durch primäre Antikörper gezeigt wurde. Während der epithelialen Wundheilung können diese mesothelialen Stammzellen beeinflusst werden, um ihre normale Heilungsfähigkeit mit Fibroblastendifferenzierung zu erhalten, anstatt gegebenenfalls ihren Phänotyp zu verändern, Proliferation auszulösen und dadurch dauerhafte Adhäsionen zu induzieren [5].

\section{Weitere Schritte}

Das Ziel zukünftiger Forschung wird es sein, die potenziell klinisch relevante Adhäsionsbildung nach einem iatrogen induzierten chirurgischen Trauma zu hemmen. Hierbei sollen Gel-inkorporierte, stammzellmodulierende Medikamente zum Einsatz kommen, um die intraabdominellen Wunden abzudecken.

\section{Interessenkonflikt}

Die Autoren geben an, dass kein Interessenkonflikt besteht.

\section{Autorinnen/Autoren}

\section{Rudy Leon De Wilde}

Klinik für Frauenheilkunde, Geburtshilfe und Gynäkologische Onkologie, Universitätsklinik für Gynäkologie, Pius-Hospital Oldenburg, Carl von Ossietzky Universität Oldenburg

\section{Lasse Leicher}

Klinik für Frauenheilkunde, Geburtshilfe und Gynäkologische Onkologie, Universitätsklinik für Gynäkologie, Pius-Hospital Oldenburg, Carl von Ossietzky Universität Oldenburg

\section{Luz Angela Torres-de la Roche}

Klinik für Frauenheilkunde, Geburtshilfe und Gynäkologische Onkologie, Universitätsklinik für Gynäkologie, Pius-Hospital Oldenburg, Carl von Ossietzky Universität Oldenburg

\section{Cristina Cezar}

Klinik für Frauenheilkunde, Geburtshilfe und Gynäkologische Onkologie, Universitätsklinik für Gynäkologie, Pius-Hospital Oldenburg, Carl von Ossietzky Universität Oldenburg

\section{Augustinus Bader}

Biomedizinisches und biotechnologisches Zentrum (BBZ) Universität Leipzig

\section{Korrespondenzadresse}

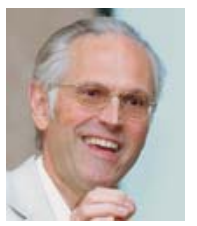

Prof. Dr. Dr. med. Rudy Leon De Wilde Klinik für Frauenheilkunde, Geburtshilfe und Gynäkologische Onkologie Universitätsklinik für Gynäkologie

Pius-Hospital Oldenburg

Carl von Ossietzky Universität Oldenburg Georgstraße 12

26121 Oldenburg

rudy-leon.dewilde@pius-hospital.de

\section{Literatur}

[1] Majewski WD. Long-term outcome, adhesions, and quality of life after laparoscopic and open surgical therapies for acute abdomen. Follow-up of a prospective trial. Surg Endosc 2005; 19: 81-90

[2] Lower AM, Hawthorn RJ, Clark D et al.; Surgical and Clinical Research (SCAR) Group. Adhesion-related readmissions following gynaecological laparoscopy or laparotomy in Scotland: an epidemiological study of 24046 patients. Hum Reprod 2004; 19: 1877-1885

[3] Herrmann A, De Wilde RL. Adhesions are the major cause of complications in operative gynecology. Best Pract Res Clin Obstet Gynaecol 2016; 35: 71-83

[4] Giri P, Ebert S, Braumann UD et al. Skin regeneration in deep second-degree scald injuries either by infusion pumping or topical application of recombinant human erythropoietin gel. Drug Des Devel Ther 2015; 9: 2565-2579

[5] Ojeh N, Pastar I, Tomic-Canic M et al. Stem cells in skin regeneration, wound healing, and their clinical applications. Int J Mol Sci 2015; 16: 25476-25501

\section{Bibliografie}

DOI https://doi.org/10.1055/a-0575-1387 Geburtsh Frauenheilk 2018; 78: 225 @ Georg Thieme Verlag KG Stuttgart . New York I ISSN 0016-5751

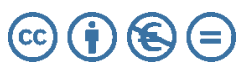

